\title{
Pregnancy and neonatal outcomes among a cohort of HIV-infected women in a large Italian teaching hospital: a 30-year retrospective study
}

\author{
S. GRIGNOLO ${ }^{1}+$, R. AGNELLO ${ }^{2} \uparrow$, D. GERBALDO ${ }^{2}$, C. GOTTA $^{3}$, C. ALICINO $^{4}$, \\ F. DEL PUENTE ${ }^{1}$, L. TARAMASSO ${ }^{1}$, B. BRUZZONE ${ }^{5}$, C. GUSTAVINO ${ }^{2}$, \\ S. TRASINO ${ }^{3}$, A. DE MARIA ${ }^{1}$, G. ICARDI ${ }^{4}$, C. VISCOLI $^{1}$ AND A. DI BIAGIO ${ }^{1}+$ \\ FOR GENOVA HIV NEONATAL AND PREGNANCY WORKING GROUP \\ ${ }^{1}$ Infectious Diseases Unit, IRCCS AOU San Martino-IST, University of Genoa, L.go Rosanna Benzi 10, 16132 \\ Genoa, Italy \\ ${ }^{2}$ Department of Obstetrics and Gynaecology, IRCCS AOU San Martino-IST, University of Genoa, L.go \\ Rosanna Benzi 10, 16132 Genoa, Italy \\ ${ }^{3}$ Neonatal Unit, IRCCS AOU San Martino-IST, L.go Rosanna Benzi 10, 16132 Genoa, Italy \\ ${ }^{4}$ Department of Health Sciences, University of Genoa, Via A. Pastore 1, 16132, Genoa, Italy \\ ${ }^{5}$ Hygiene Unit, IRCCS AOU San Martino-IST, L.go Rosanna Benzi 10, 16132 Genoa, Italy
}

Received 6 September 2016; Final revision 14 February 2017; Accepted 22 February 2017; first published online 22 March 2017

\section{SUMMARY}

The primary study objective was to investigate three decades from 1985 to 2014 of changes in pregnancies among HIV-infected women. The secondary objective was to assess risk factors associated with preterm delivery and severe small-for-gestational-age (SGA) infants in HIVinfected women. A retrospective review of deliveries among pregnant HIV-infected women at the University of Genoa and IRCCS San Martino-IST in Genoa between 1985 and 2014 was performed. Univariate and multivariable analyses were used to study the variables associated with neonatal outcomes. Overall, 262 deliveries were included in the study. An increase in median age (26 years in 1985-1994 vs. 34 years in 2005-2014), in the proportion of foreigners (none in 1985-1994 vs. 27/70 (38.6\%) in 2005-2014), and a decrease in intravenous drug use (75.2\% (91/121) in 1985-1994 vs. 12.9\% (9/70) in 2005-2014) among pregnant HIV-infected women was observed. Progressively, HIV infections were diagnosed sooner (prior to pregnancy in $80 \%(56 / 70)$ of women in the last decade). An increase in combined antiretroviral therapy (cART) prescription during pregnancy (50\% (27/54) in 1995-2004 vs. 92.2\% (59/64) in 20052014) and in HIV-RNA <50 copies/ml at delivery (19.2\% (5/26) in 1995-2004 vs. 82.3\% (53/64) in 2005-2014) was observed. The rate of elective caesarean section from 1985 to 1994 was $9 \cdot 1 \%$, which increased to $92 \cdot 3 \%$ from 2004 to 2015 . Twelve (10.1\%) mother-to-child transmissions (MTCT) occurred in the first decade, and six (8.3\%) cases occurred in the second decade, the last of which was in 2000. Preterm delivery (<37 weeks gestation) was 5\% (6/121) from 1985 to 1994 and increased to $17 \cdot 1 \%(12 / 70)$ from 2005 to 2014. In univariate and multivariable logistic regression analyses, advancing maternal age and previous pregnancies were associated with preterm delivery (odds ratio (OR) 2.7; 95\% confidence intervals (CI) $1-7 \cdot 8$ and OR 2.6; $95 \%$ CI

\footnotetext{
* Authors for correspondence: S. Grignolo, Infectious Diseases Unit, IRCCS AOU San Martino - IST, University of Genoa, L.go R. Benzi 10, 16132, Genoa, Italy.

(Email: sara.grignolo@gmail.com)

$\uparrow \mathrm{SG}$ and RA contributed equally in the paper.

* Members of the Genova HIV Neonatal and Pregnancy Working Group are listed in the acknowledgment section.
} 
$1 \cdot 1-6 \cdot 7$, respectively). In the logistic regression analysis, use of heroin or methadone was found to be the only risk factor for severe SGA (OR $3 \cdot 1 ; 95 \%$ CI 1.4-6.8). In conclusion, significant changes in demographic, clinical and therapeutic characteristics of HIV-infected pregnant women have occurred over the last 30 years. Since 2000, MTCT has decreased to zero. An increased risk of preterm delivery was found to be associated with advancing maternal age and previous pregnancies but not with cART. The use of heroin or methadone has been confirmed as a risk factor associated with severe SGA.

Key words: HIV, mother-to-child transmission, pregnancy, preterm delivery, small for gestational age.

\section{INTRODUCTION}

Recommendations regarding the management of HIV-infected women during pregnancy have evolved considerably in Europe in recent years, reflecting changes in the epidemic, as well as in the knowledge of HIV prevention and treatment [1]. In the USA, the overall annual rate of mother-to-child transmission (MTCT) of HIV decreased from 3\% to $2 \%$ from 2005 to 2008 [2]. In the UK and Ireland, MTCT rates have continued to decline since 2006, reaching an all-time low of $0.46 \%$ in 2010-2011 [3]. In Italy, between 2013 and 2014, only nine children acquired an HIV infection during pregnancy, labour or breastfeeding [4]. Nevertheless, debates about several issues such as the overuse of elective caesarean deliveries remain open, particularly regarding women who have plasma HIV-RNA < 50 copies/ml and no other contraindication to a vaginal delivery [5]. Current guidelines recommend a caesarean section only when the mother has a detectable HIV-RNA [5], since it can result in a potentially useless and harmful intervention when the maternal viral load is undetectable [6]. Moreover, the incidence and frequency of adverse pregnancy outcomes, in particular in women receiving antiretroviral therapy (ART), might require further investigation. Finally, recent changes in HIV epidemiology and their potential influence on engagement in care, therapy adherence and retention in care are of particular interest, along with the sociodemographic and lifestyle factors of these patients [7]. In this context, the primary changes recorded in Italy within the last years have been characterised by an increase in the number of foreign citizens diagnosed with HIV and a change in risk factors for infection (i.e., an increase in heterosexual transmission in women compared with the one caused by intravenous drug use) [4]. Moreover, Italian women are now the oldest in Europe at the time of their first pregnancy [8].
The primary objective of the study was to analyse trends and patterns of pregnancies among HIVinfected women over three decades in a 'real-life' setting of care. A secondary objective was to assess the risk factors associated with preterm delivery and severe small-for-gestational-age (SGA) infants in HIV-infected women. This observational, longitudinal study includes the full database of deliveries among HIV-infected women from 1985 to 2014 that occurred at University of Genoa and IRCCS San Martino-IST in Genoa, Italy.

\section{METHODS}

A retrospective review of deliveries among HIVinfected women followed at the University of Genoa and IRCCS San Martino-IST in Genoa between January 1985 and December 2014 was performed. The study population includes women from the European Collaborative Study (ECS), a prospective cohort study enrolling HIV-positive pregnant women in 10 European countries in 1985-2011, and women excluded from the ECS. Our research centre enrolled HIV-positive women in ECS from 1985 to 2004. Therefore, our cohort of HIV-infected pregnant women was composed of women enrolled in the ECS in 1985-2004, women excluded from the ECS because they did not fulfil the eligibility criteria (pregnant women who received HIV diagnosis before or during pregnancy) and women with pregnancy in 2005-2014. The informed consent of women enrolled in ECS was obtained before their enrolment, whereas informed consent of other women was not required, as this is a clinical audit of routinely collected clinical data. However, informed consent for the use of anonymised data for scientific purposes was signed by all patients admitted to IRCCS AOU San MartinoIST and included in the study. A consent from the Ethical Committee of Liguria Region was obtained 
for data analysis and publication purposes. According to the ECS protocol [9], the collected data included demographic characteristics, HIV infection, hepatitis $\mathrm{C}$ virus (HCV) and hepatitis $\mathrm{B}$ virus (HBV) co-infection, heroin and methadone use during pregnancy, plasma HIV-RNA load at delivery, ART before and during pregnancy, antiretroviral prophylaxis during labour and delivery, and mode of delivery. Information on preterm delivery, birth weight, mode of infant feeding and HIV infection were also obtained for each newborn. The analyses were based on the number of pregnancies rather than women, thus some women were included more than once.

Years of delivery were grouped in three decades: 1985-1994, 1995-2004 and 2005-2014. The timing of HIV diagnosis was classified as it occurred: (a) prior to pregnancy, in women already known to be HIV infected; (b) during pregnancy, in women screened for HIV infection during pregnancy and resulted HIV positive; (c) at/after delivery, in women not screened for HIV during pregnancy but only at or immediately after delivery. In term of exposure to ART, pregnancies were classified as untreated, treated with monotherapy or dual therapy, and receiving combined antiretroviral therapy (cART). cART was categorised as protease inhibitor (PI)-based regimen or else, based on the PIs inclusion or exclusion. $\mathrm{CD}^{+}{ }^{+} \mathrm{T}$-cell count (not routinely reported before 2000) was categorised as $<200$ cells $/ \mu 1,200-499$ cells/ $\mu \mathrm{l}$ and $\geqslant 500 \mathrm{cell} / \mu \mathrm{l}$. The latest $\mathrm{CD}^{+}{ }^{+} \mathrm{T}$-cell count before delivery was included in the analysis. HIV-1 RNA levels were determined through the following assays: Nuclisens EasyQ HIV-1 2·0 (BioMérieux SA, Marcy l'Etoile, France). Tests were usually carried out on plasma samples, but occasionally on stored serum samples. HIV-RNA (collected from 1998 onward) was categorised as undetectable ( $\leqslant 50 \mathrm{cop}$ ies $/ \mathrm{ml}$ ) or detectable ( $>50$ copies $/ \mathrm{ml}$ ), according to the cut-off value defining virological suppression reported in European and Italian Guidelines [10, 11]. The closest to delivery HIV-RNA value was used in the analysis.

Mode of delivery was reported as vaginal or per caesarean section. Caesarean section was defined as elective when performed before the onset of labour and with intact membranes, and as emergency in the other cases. Delivery was defined as preterm when it occurred before the 37th week of gestation. A severe SGA birth was identified with a newborn birth weight lower than the 3rd percentile for the gestational age [12]. A narrow definition of SGA was used because infants with birth weight lower than 3rd percentile were strongly and most consistently associated with adverse perinatal outcome [13]. HIV status in the infant was investigated as follows: at birth, at 2-4-6 weeks of age and at 3-6-9-12-18-24 months of age (as the infant was still positive at 18 month) using both serological and molecular assays. For the serological follow-up, Vironostika HIVAg/Ab UniForm EIA (BioMérieux Laboratories, Boxtel, NL, USA), Genscreen Plus HIV Ag/Ab EIA (Bio-rad, Marne la Coquette, France) and New Lav Blot I (Bio-rad, Marne la Coquette, France) were performed. For the molecular follow-up, HIV-1 RNA and HIV-1 DNA in PBMC (peripheral blood mononuclear cells) were performed. HIV-RNA was measured by using Nuclisens EasyQ HIV-1 2.0 (BioMérieux SA, Marcy l'Etoile, France). HIV-1 DNA was measured by using the C-PCR (commercial PCR) system manufactured by Amplimedical S.p.A - Bioline Division (Italy), designed to amplify a 600-base pair (bp) undefined pol region since the test was available on the market; subsequently, HIV-1 DNA was performed by using a H-PCR (homemade PCR) system designed to amplify a highly conserved $508 \mathrm{bp} \mathrm{HIV-1}$ pol integrase region [14]. HIV infection was excluded if HIV serological and molecular assays were negative.

\section{Statistical analysis}

All statistical analyses were performed using Epi-Info 7.0 (Centers for Disease Control and Prevention (CDC), Atlanta, GA, USA) and JMP ${ }^{\circledR} 10$ (SAS Institute Inc., Cary, NC, USA) software for Windows.

Continuous numeric variables were summarised as mean and standard deviation for normalised distributed variables and as median and interquartile range for non-normalised variables. Categorical variables were summarised in the form of percentage proportions.

The baseline characteristics of the study population by decade of pregnancy were compared using $\chi^{2}$ and Fisher exact test [15] and the differences in numeric variables were evaluated through ANOVA (analysis of variance) test for normalised distributed variables or Wilcoxon signed-rank test for non-parametric statistical analysis as appropriate [16].

For the assessment of factors associated with preterm birth and severe SGA, continuous variables were dichotomised and cut-off values were determined through ROC (receiver operating characteristic) curves. Then, all variables were compared in 
univariate analyses by the $\chi^{2}$ test or the Fisher exact test, as appropriate. These tests were performed for both comparisons and they all were two-sided. To assess the independent role of risk factors, variables with a $P$ value $<0.05$ in univariate comparisons were included in logistic regression models. The odds ratio (OR), 95\% confidence intervals $(95 \% \mathrm{CI})$ and corresponding $P$ values obtained in the logistic regression models were reported.

All tests were two-tailed and a $P$ value $<0.05$ was determined to represent statistical significance.

\section{RESULTS}

Our cohort included 262 deliveries from 231 HIV-infected women. One hundred and twenty-one deliveries (46-2\%) occurred in the decade 1985-1994, 71 deliveries $(27 \cdot 1 \%)$ occurred in the decade 1995 2004 and 70 deliveries $(26.7 \%)$ within the period 2005-2014. Of these deliveries, $143(54 \cdot 6 \%)$ were in women enrolled in the ECS and 119 were in women not enrolled in ECS because they were diagnosed with HIV at or after their delivery $(49,18.7 \%)$ or they became pregnant after 2004 (70, 26.7\%). The demographic and clinical characteristics of the women, stratified by decade, are listed in Table 1. Briefly, in the first decade all women were Italian, their median age was 26 years, and drug injection was the most frequent risk factor for HIV infection $(91 / 121,75 \cdot 2 \%)$. Twenty-four out of 121 patients $(19 \cdot 8 \%)$ were HCV-infected, 50/121 women $(41 \cdot 3 \%)$ were diagnosed prior to their pregnancy and 28/121 women $(23 \cdot 2 \%)$ were diagnosed during their pregnancy. In the last decade, $27 / 70$ women (38.6\%) were not Italian, the median age was 34 years, $44 / 70$ patients $(62.9 \%)$ had heterosexual intercourse as a risk category for HIV infection and 7/70 women $(10 \%)$ were HCV co-infected. The diagnosis of HIV infection was performed prior to pregnancy in the majority of cases $(56 / 70,80 \%)$. Conversely, among the foreigners the median age was 31 years for African women and 36 years for South-American women, and the majority of them had heterosexual intercourse as a risk category for HIV infection (21/ $22,91.5 \%$ of South-American women and 6/11, $54.5 \%$ of African women), and HCV co-infection was uncommon (1/22, 4.5\% among South-American women and $0 \%$ among African women) (Supplementary Table S1 in the Supplementary Material). Almost all of the differences in demographic and clinical characteristics analysed according to calendar year and ethnic group were statistically significant based on univariate analysis $(P<0.001$, for all the characteristics noted above). In the first decade, all patients were not on ART at conception and almost all (107/ $121,88 \cdot 4 \%$ ) had not been treated during pregnancy. All 14 patients treated with ART in this period received monotherapy. The proportion of patients on ART at conception and during pregnancy was significantly higher in foreign women compared with Caucasian women $(19 / 22,86 \cdot 4 \%$ and $9 / 22,40 \cdot 9 \%$ among South-American women and $9 / 11,81.8 \%$ and $4 / 11,36 \cdot 4 \%$ among African women, respectively), and increased during the following decades $(9 / 71,12.7 \%$ and $36 / 71,76 \cdot 1 \%$ in the second decade, $36 / 70,51 \cdot 4 \%$ and $64 / 70,91.4 \%$ in the third decade, respectively, $P<0.001)$, as well as the proportion of women receiving cART $(27 / 71,50 \%$ in the second decade, $59 / 70,92.2 \%$ in the third decade, $P<0.001$ ). Demographic and clinical characteristics of HIV-infected pregnant women according to use of methadone or heroin are listed in Supplementary Table S2 in the Supplementary Material. Information related to $\mathrm{CD} 4^{+}$T-cell count and plasma HIV-RNA load at delivery was available only for a small number of women $(91 / 262,35 \%$ and 90/262, 34\%, respectively). The proportion of women with HIV-RNA load $\leqslant 50$ copies/ml at delivery was significantly higher in recent years $(5 / 26,19 \cdot 2 \%$ in $1995-2004$ vs. $53 / 64$, $82 \cdot 3 \%$ in $2005-2014, P<0 \cdot 001)$.

In the first decade, vaginal delivery was the most frequent mode of delivery $(107 / 121,88 \cdot 4 \%)$, while elective caesarean sections progressively increased in the following years $(11 / 121,9 \cdot 1 \%$ in $1985-1994$; 44/ $71,63 \cdot 7 \%$ in $1995-2004$; $65 / 70,92 \cdot 9 \%$ in $2005-2014$, $P<0.001)$. In the last decade, no women delivered vaginally, $65 / 70(92.9 \%)$ women had an elective caesarean section and 5/70 (7.1\%) women had an emergency caesarean section. Of the women who received an elective caesarean section, 55/84 (65.5\%) had an undetectable plasma HIV-RNA load at delivery (Supplementary Table S3 in the Supplementary Material).

The number of twin pregnancies was one in the first decade, one in the second decade and two in the third decade. The rate of preterm delivery was significantly higher in the last decade $(6 / 121,5.0 \%$ in $1985-1994$; $7 / 71,9 \cdot 8 \%$ in $1995-2004 ; 12 / 70,17 \cdot 1 \%$ in $2005-2014$, $P=0.02)$. Severe SGAs were similar in the three periods $(23 / 121,19 \cdot 5 \%$ in $1985-1994 ; 21 / 71,29 \cdot 6 \%$ in $1995-2004 ; 11 / 70,15 \cdot 7 \%$ in $2005-2014 ; P=0 \cdot 11$ ). Four infants were characterised by both preterm 
Table 1. Demographic and clinical characteristics of HIV-infected pregnant women and neonatal outcome according to year of delivery*

\begin{tabular}{|c|c|c|c|c|c|}
\hline & $\begin{array}{l}\text { Total } \\
(N=262) \\
n / N(\%)\end{array}$ & $\begin{array}{l}1985-1994 \\
(N=121) \\
n / N(\%)\end{array}$ & $\begin{array}{l}1995-2004 \\
(N=71) \\
n / N(\%)\end{array}$ & $\begin{array}{l}2005-2014 \\
(N=70) \\
n / N(\%)\end{array}$ & $P$ value \\
\hline Total deliveries & 262 & 121 & 71 & 70 & \\
\hline Median age (IQR) & $28(25-34)$ & $26(23-28)$ & $31(28-36)$ & $34(29-37)$ & $<0 \cdot 001$ \\
\hline Ethnic group & & & & & $<0 \cdot 001$ \\
\hline Caucasian & $227 / 262(86 \cdot 6)$ & $121 / 121(100)$ & $63 / 71(88 \cdot 7)$ & $43 / 70(61 \cdot 4)$ & \\
\hline Not Caucasian & $35 / 262(13 \cdot 4)$ & $0 / 121(0)$ & $8 / 71(11 \cdot 3)$ & $27 / 70(38 \cdot 6)$ & \\
\hline South-American & $22 / 35(62.9)$ & - & $6 / 8(75)$ & $16 / 27(59 \cdot 3)$ & \\
\hline African & $11 / 35(31 \cdot 4)$ & - & $2 / 8(25)$ & $9 / 27(33 \cdot 3)$ & \\
\hline Asian & $2 / 35(5 \cdot 7)$ & - & $0 / 8(0)$ & $2 / 27(7 \cdot 4)$ & \\
\hline \multicolumn{6}{|l|}{ HIV risk factors (mode of transmission) } \\
\hline Injecting drugs use & $127 / 262(48 \cdot 5)$ & $91 / 121(75 \cdot 2)$ & $27 / 71(38)$ & $9 / 70(12 \cdot 9)$ & $<0 \cdot 001$ \\
\hline Heterosexual intercourse & $99 / 262(37 \cdot 8)$ & $22 / 121(18 \cdot 2)$ & $33 / 71(46 \cdot 5)$ & $44 / 70(62 \cdot 9)$ & $<0 \cdot 001$ \\
\hline MTCT & $3 / 262(1 \cdot 1)$ & $0 / 121(0)$ & $0 / 71(0)$ & $3 / 70(4 \cdot 3)$ & $0 \cdot 02$ \\
\hline Other & $33 / 262(12 \cdot 6)$ & $8 / 121(6 \cdot 6)$ & $11 / 71(15 \cdot 5)$ & $14 / 70(20)$ & $0 \cdot 02$ \\
\hline \multicolumn{6}{|l|}{ Co-infection } \\
\hline HCV co-infection & $60 / 262(22 \cdot 9)$ & $24 / 121(19 \cdot 8)$ & $29 / 71(40 \cdot 8)$ & $7 / 70(10)$ & $<0 \cdot 001$ \\
\hline HBV co-infection & $32 / 262(12 \cdot 2)$ & $18 / 121(56 \cdot 3)$ & $11 / 71(15 \cdot 4)$ & $3 / 70(4 \cdot 3)$ & $0 \cdot 06$ \\
\hline HBV and HCV co-infection & $16 / 262(6 \cdot 1)$ & $5 / 121(4 \cdot 1)$ & $9 / 71(12 \cdot 7)$ & $2 / 70(2 \cdot 9)$ & $0 \cdot 02$ \\
\hline Smoke & $53 / 262(20 \cdot 2)$ & 29/121 (24) & $17 / 71(24)$ & $7 / 70(10)$ & $0 \cdot 04$ \\
\hline Methadone or heroin & $39 / 262(14 \cdot 9)$ & $25 / 121(20 \cdot 6)$ & $11 / 71(15 \cdot 5)$ & $3 / 70(4 \cdot 3)$ & $0 \cdot 009$ \\
\hline Timing of HIV diagnosis & & & & & $<0 \cdot 001$ \\
\hline Prior to pregnancy & $161 / 262(61 \cdot 5)$ & $50 / 121(41 \cdot 3)$ & $55 / 71(77 \cdot 5)$ & $56 / 70(80)$ & \\
\hline During pregnancy & $48 / 262(18 \cdot 3)$ & $28 / 121(23 \cdot 2)$ & $10 / 71(14 \cdot 1)$ & $10 / 70(14 \cdot 3)$ & \\
\hline At/after delivery & $53 / 262(20 \cdot 2)$ & $43 / 121(35 \cdot 5)$ & $6 / 71(8 \cdot 4)$ & $4 / 70(5 \cdot 7)$ & \\
\hline ART at conception & $45 / 262(17 \cdot 2)$ & $0 / 121(0)$ & $9 / 71(12 \cdot 7)$ & $36 / 70(51 \cdot 4)$ & $<0 \cdot 001$ \\
\hline ART prescription during pregnancy & $132 / 262(50 \cdot 4)$ & $14 / 121(11 \cdot 6)$ & $54 / 71(76 \cdot 1)$ & $64 / 70(91 \cdot 4)$ & $<0 \cdot 001$ \\
\hline Monotherapy/dual therapy & $46 / 132(34 \cdot 8)$ & $14 / 14(100)$ & $27 / 54(50)$ & $5 / 70(7 \cdot 8)$ & $<0 \cdot 001$ \\
\hline cART & $86 / 132(65 \cdot 2)$ & $0 / 14(0)$ & $27 / 54(50)$ & $59 / 70(92 \cdot 2)$ & 0.005 \\
\hline$c A R T$ protease inhibitor based & $67 / 86(50 \cdot 8)$ & - & $16 / 27(29 \cdot 6)$ & $51 / 59(79 \cdot 7)$ & \\
\hline Mode of delivery $(n=260)$ & & & & & $<0 \cdot 001$ \\
\hline Vaginal & $127 / 260(48 \cdot 8)$ & $107 / 121(88 \cdot 4)$ & $20 / 69(29)$ & $0 / 70(0)$ & \\
\hline Elective caesarean section & $120 / 260(46 \cdot 2)$ & $11 / 121(9 \cdot 1)$ & $44 / 69(63 \cdot 7)$ & $65 / 70(92 \cdot 9)$ & \\
\hline Emergency caesarean section & $13 / 260(5)$ & $3 / 121(2 \cdot 5)$ & $5 / 69(7 \cdot 3)$ & $5 / 70(7 \cdot 1)$ & \\
\hline Antiretroviral prophylaxis intrapartum & $117 / 262(44 \cdot 7)$ & $0 / 121(0)$ & $51 / 71(71 \cdot 8)$ & $66 / 70(94 \cdot 3)$ & $<0 \cdot 001$ \\
\hline HIV-RNA load at delivery ${ }^{\dagger}(n=90)$ & & & & & $<0 \cdot 001$ \\
\hline Undetectable $(\leqslant 50 \mathrm{cp} / \mathrm{ml})$ & $58 / 90(64 \cdot 4)$ & - & $5 / 26(19 \cdot 2)$ & $53 / 64(82 \cdot 8)$ & \\
\hline Detectable $(>50 \mathrm{cp} / \mathrm{ml})$ & $32 / 90(35 \cdot 6)$ & - & $21 / 26(80 \cdot 8)$ & $11 / 64(17 \cdot 2)$ & \\
\hline $\mathrm{CD}^{+}{ }^{+}$T-cell at delivery ${ }^{\dagger}(n=91)$ & & & & & $0 \cdot 005$ \\
\hline$<200 \mathrm{cell} / \mathrm{mm}^{3}$ & $3 / 91(3 \cdot 3)$ & $1 / 5(20)$ & $1 / 26(3 \cdot 8)$ & $1 / 60(1 \cdot 6)$ & \\
\hline $200-499 \mathrm{cell} / \mathrm{mm}^{3}$ & $29 / 91(31 \cdot 9)$ & $3 / 5(60)$ & $13 / 26(50)$ & $13 / 60(21 \cdot 7)$ & \\
\hline$\geqslant 500 \mathrm{cell} / \mathrm{mm}^{3}$ & $59 / 91(64 \cdot 8)$ & $1 / 5(20)$ & $12 / 26(46 \cdot 2)$ & $46 / 60(76 \cdot 7)$ & \\
\hline Previous pregnancy & $93 / 262(35 \cdot 5)$ & $30 / 121(24 \cdot 7)$ & $29 / 71(40 \cdot 9)$ & $34 / 70(48 \cdot 6)$ & $0 \cdot 002$ \\
\hline Previous pregnancy interruption & $99 / 262(37 \cdot 8)$ & $54 / 121(44 \cdot 6)$ & $27 / 71(38)$ & $18 / 70(25 \cdot 7)$ & $0 \cdot 03$ \\
\hline Previous spontaneous abortion & $29 / 262(11 \cdot 1)$ & $14 / 121(11 \cdot 6)$ & $7 / 71(9 \cdot 9)$ & $8 / 70(11 \cdot 4)$ & $0 \cdot 92$ \\
\hline PROM & $95 / 262(36 \cdot 3)$ & $73 / 121(60 \cdot 3)$ & $17 / 71(23 \cdot 9)$ & $5 / 70(7 \cdot 1)$ & $<0 \cdot 001$ \\
\hline Preterm delivery & $25 / 262(9 \cdot 5)$ & $6 / 121(5)$ & $7 / 71(9 \cdot 8)$ & $12 / 70(17 \cdot 1)$ & $0 \cdot 02$ \\
\hline Severe $\mathrm{SGA}^{\S}$ & $55 / 262(21)$ & $23 / 121(19 \cdot 5)$ & $21 / 71(29 \cdot 6)$ & $11 / 70(15 \cdot 7)$ & $0 \cdot 11$ \\
\hline Infant's HIV infection $(n=263)^{\top}$ & $18 / 263(6 \cdot 8)$ & $12 / 119(10 \cdot 1)$ & $6 / 72(8 \cdot 3)$ & $0 / 72(0)$ & $0 \cdot 02$ \\
\hline
\end{tabular}

IQR, interquartile range; MTCT, mother-to-child transmission; HBV, hepatitis B virus; HCV, hepatitis C virus; ART, antiretroviral therapy; cART, combined antiretroviral therapy; PROM, premature rupture of membranes; SGA, small for gestational age.

* Percentages exclude unknown/missing results.

$\dagger$ Measurement closest to delivery.

+ Delivery was defined as preterm when it occurred before 37 th gestational week.

${ }^{\$}$ Severe SGA birth was defined when newborn birth weight was lower than the 3rd percentile for the gestational age.

"In the first decade, there were three deaths in newborns after delivery and one twin pregnancy. One twin pregnancy was in the second and two were in the third decade. 
delivery and severe SGA birth. Only three infants were breastfed (2/121 in 1985-1994 and 1/71 in 1995-2004). In the first decade, three newborns died after delivery and their serological status was unknown. Overall, 18 newborns $(6 \cdot 8 \%)$ were HIV-infected, all in the period 1985-2004. Of these newborns, $12(10 \cdot 1 \%)$ were born in the first decade and $6(8 \cdot 3 \%)$ were born in the second decade. The last case of MTCT was observed in 2000 .

The results of the univariate analysis and multivariable logistic regression analysis for the preterm deliveries are listed in Table 2. An advanced maternal age (age $\geqslant 32$ years) and previous pregnancies were the maternal factors associated with preterm delivery (OR 2.9; 95\% CI 1.1-8.4; $P=0.03$ and OR 2.5; 95\% CI $1-6.4 ; P<0.05$, respectively). The univariate and multivariable logistic regression analyses for severe SGA infants are reported in Table 3. Among the maternal risk factors explored, only the maternal use of heroin or methadone during pregnancy was correlated with severe SGA infants (OR 3.1; 95\% CI 1.4$6 \cdot 8 ; P=0 \cdot 007)$.

\section{DISCUSSION}

In this cohort of HIV-infected pregnant women from a large teaching hospital in Genoa, we described the changes in demographic, clinical and therapeutic characteristics and neonatal outcomes that occurred in the decades 1985-2014. In short, we report a significant increase in the median age (currently 34 years) and in the proportion of foreigners among HIV-infected pregnant women (currently 38.6\%). Furthermore, in the last decade the most frequent risk factor for HIV infection in pregnant women has shifted away from injected drug use to heterosexual intercourse, and vertical transmission has become another risk category for HIV infection among pregnant women. With the reduction in injecting drug use as a risk factor, the number of HCV co-infected pregnant women decreased as well from $19 \cdot 8 \%$ in 1985-1994 to $10 \%$ in 2005-2014, as observed among the HIV-infected general population [17]. In recent years, the proportion of patients on ART at conception and during pregnancy significantly increased, as expected. In fact, in the last decade $51.4 \%$ of women were on ART at conception and $91.4 \%$ received treatment during their pregnancy. Among antiretroviral regimens, cART has become the most widely used $(92.2 \%)$, achieving the suppression of HIV-RNA load at delivery in the majority of cases $(82 \cdot 8 \%)$.
These changes in the epidemiology of HIV-infected pregnant women reported in our cohort are consistent with surveillance data and findings of previously published studies [17-21]. In a pooled analysis of data on HIV-infected pregnant women enrolled in the Swiss Mother \& Child HIV Cohort Study and the ECS between 2000 and 2010, Aebi-Popp et al. reported data similar to ours: overall, $48 \%$ women were black, the most common mode of HIV acquisition was heterosexual contact $(76 \%), 16 \%$ of women had a history of injecting drug use, the majority of pregnant women were aware of their HIV diagnosis before conception (72\%), 91\% received ART throughout their pregnancy and delivery and $86 \%$ had an undetectable HIV-RNA load at delivery [20]. In our cohort, foreign women were older than Italian women (median age, 31 years for African women, 36 years for South-American women vs. 28 years for Italian women). Moreover, they were more likely to be receiving ART at conception and a higher proportion of them received cART during pregnancy compared with Caucasian women. Even though the fraction of foreigners has been increasing in the recent years, overall they represented only $13.4 \%$ of the women in our cohort.

The prevalence of elective caesarean sections progressively increased within the last few years to become the most frequent mode of delivery $(92.9 \%$ elective and $7 \cdot 1 \%$ emergency caesarean sections). The very high rate of elective caesarean section deliveries reported in our study is not surprising considering that Italy is the country with the highest rate of caesarean sections among HIV-uninfected women in the European Union [22]. We note, however, that this situation contradicts European and national guidelines [10, 11, 23]. Elective caesarean sections have been recommended to prevent MTCT in the absence of ART since 1997 $[24,25]$. However, with the growing use of cART the additional benefit of an elective caesarean section in reducing MTCT became uncertain; European and national guidelines changed and now recommend vaginal deliveries for all HIV-infected women with an undetectable HIV-RNA load at delivery in the absence of an obstetric indication for an elective caesarean section. We reported 'real-life' data in which the high rate of elective caesarean sections could be partially justified and may have contributed to reducing MTCT, considering that during the last decade $5.7 \%$ of HIV diagnoses in our cohort still occurred at/after delivery, only $91.4 \%$ of pregnant women received cART during pregnancy and $17 \cdot 2 \%$ of 
Table 2. Univariate and multivariable logistic regression analysis for preterm delivery $(<37$ completed weeks gestation $)^{+}$

\begin{tabular}{|c|c|c|c|c|c|c|}
\hline & \multirow{2}{*}{$\begin{array}{l}\text { No preterm } \\
(N=237) \\
n / N(\%)\end{array}$} & \multirow{2}{*}{$\begin{array}{l}\text { Preterm } \\
(N=25) \\
n / N(\%)\end{array}$} & \multicolumn{2}{|c|}{ Univariate analysis } & \multicolumn{2}{|c|}{ Multivariable analysis* } \\
\hline & & & OR (CI 95\%) & $P$ value & $\begin{array}{l}\text { Adjusted OR } \\
\text { (CI 95\%) }\end{array}$ & $P$ value \\
\hline Decade of birth & & & & $0 \cdot 02$ & & $0 \cdot 82$ \\
\hline 1985-1994 & $115 / 237(48 \cdot 5)$ & $6 / 25(24)$ & Reference & & Reference & \\
\hline 1995-2004 & $64 / 237(27)$ & $7 / 25(28)$ & $2 \cdot 1(0 \cdot 7-6 \cdot 7)$ & & $0 \cdot 8(0 \cdot 1-6 \cdot 4)$ & \\
\hline 2005-2014 & $58 / 237(24 \cdot 5)$ & $12 / 25(48)$ & $4(1 \cdot 5-11 \cdot 9)$ & & $1 \cdot 2(0 \cdot 1-11 \cdot 4)$ & \\
\hline Age $\geqslant 32$ years & $71 / 237(30)$ & $17 / 237(66 \cdot 7)$ & $4 \cdot 7(2-12)$ & $<0.001$ & $2 \cdot 7(1-7 \cdot 8)$ & $<0 \cdot 05$ \\
\hline Ethnic group & & & & $0 \cdot 83$ & & \\
\hline Caucasian & $205 / 237(86 \cdot 5)$ & $22 / 237(88)$ & Reference & & & \\
\hline Not Caucasian & $32 / 237(13 \cdot 5)$ & $3 / 237(12)$ & $0 \cdot 9(0 \cdot 2-2 \cdot 7)$ & & & \\
\hline \multicolumn{7}{|c|}{ HIV risk factors (mode of transmission) } \\
\hline Injecting drugs use & $114 / 237(48 \cdot 1)$ & $13 / 25(52)$ & $1 \cdot 2(0 \cdot 5-2 \cdot 7)$ & $0 \cdot 71$ & & \\
\hline Heterosexual intercourse & $90 / 237(38)$ & $9 / 25(36)$ & $0 \cdot 9(0 \cdot 4-2 \cdot 1)$ & $0 \cdot 85$ & & \\
\hline MTCT & $3 / 237(1 \cdot 3)$ & $0 / 25(0)$ & $2 \cdot 3(0-8 \cdot 7)$ & $>0.99$ & & \\
\hline Other & $30 / 237(12 \cdot 7)$ & $3 / 25(12)$ & $0 \cdot 9(0 \cdot 2-2 \cdot 9)$ & $0 \cdot 92$ & & \\
\hline \multicolumn{7}{|l|}{ Co-infection } \\
\hline HCV co-infection & $54 / 237(22 \cdot 8)$ & $6 / 25(24)$ & $1 \cdot 1(0 \cdot 4-2 \cdot 7)$ & $0 \cdot 89$ & & \\
\hline HBV co-infection & $31 / 237(13 \cdot 1)$ & $1 / 25(4)$ & $0 \cdot 3(0 \cdot 01-1 \cdot 4)$ & $0 \cdot 14$ & & \\
\hline $\mathrm{HBV}$ and $\mathrm{HCV}$ co-infection & $15 / 237(6 \cdot 3)$ & $1 / 25(4)$ & $0 \cdot 6(0 \cdot 03-3 \cdot 3)$ & $0 \cdot 62$ & & \\
\hline Smoke & $49 / 237(20 \cdot 7)$ & $4 / 25(16)$ & $0 \cdot 7(0 \cdot 2-2)$ & $0 \cdot 57$ & & \\
\hline Use of methadone or heroin & $34 / 237(14 \cdot 4)$ & $5 / 25(20)$ & $1 \cdot 5(0 \cdot 5-4)$ & $0 \cdot 47$ & & \\
\hline Timing of HIV diagnosis & & & & $0 \cdot 05$ & & \\
\hline Prior to pregnancy & $143 / 237(60 \cdot 3)$ & $18 / 25(72)$ & Reference & & & \\
\hline During pregnancy & $42 / 237(17 \cdot 7)$ & $6 / 25(24)$ & $1 \cdot 1(0 \cdot 4-2 \cdot 9)$ & & & \\
\hline At/after delivery & $52 / 237(21 \cdot 9)$ & $1 / 25(4)$ & $0 \cdot 2(0 \cdot 01-0 \cdot 8)$ & & & \\
\hline ART at conception & $41 / 237(17 \cdot 3)$ & $4 / 25(16)$ & $0 \cdot 9(0 \cdot 3-2 \cdot 5)$ & $0 \cdot 87$ & & \\
\hline ART prescription during pregnancy & $115 / 237(48 \cdot 5)$ & $17 / 25(68)$ & $2 \cdot 3(1-5 \cdot 7)$ & $0 \cdot 06$ & & \\
\hline Type of ART during pregnancy & & & & $0 \cdot 09$ & & $0 \cdot 86$ \\
\hline No ART & $122 / 237(51 \cdot 5)$ & $8 / 25(32)$ & Reference & & Reference & \\
\hline Monotherapy/dual therapy & $42 / 237(17 \cdot 7)$ & $4 / 25(16)$ & $1.5(0 \cdot 4-4 \cdot 9)$ & & $0 \cdot 7(0 \cdot 1-3 \cdot 5)$ & \\
\hline cART & $73 / 237(30 \cdot 8)$ & $13 / 25(52)$ & $2 \cdot 7(1 \cdot 1-7 \cdot 2)$ & & $0 \cdot 6(0 \cdot 1-3 \cdot 4)$ & \\
\hline Antiretroviral prophylaxis intrapartum & $99 / 237(41 \cdot 8)$ & $18 / 25(72)$ & $3 \cdot 6(1 \cdot 5-9 \cdot 5)$ & $0 \cdot 004$ & $2 \cdot 8(0 \cdot 4-57 \cdot 4)$ & $0 \cdot 32$ \\
\hline HIV-RNA load at delivery $(n=90)$ & & & & $0 \cdot 54$ & & \\
\hline Undetectable $(\leqslant 50 \mathrm{cp} / \mathrm{ml})$ & $50 / 76(65 \cdot 8)$ & $8 / 14(57 \cdot 1)$ & Reference & & & \\
\hline Detectable $(>50 \mathrm{cp} / \mathrm{ml})$ & $26 / 76(34 \cdot 2)$ & $6 / 14(42 \cdot 9)$ & $1 \cdot 4(0 \cdot 4-4 \cdot 6)$ & & & \\
\hline $\mathrm{CD}^{+}$T-cell at delivery* $(n=91)$ & & & & $0 \cdot 61$ & & \\
\hline$<200 \mathrm{cell} / \mathrm{mm}^{3}$ & $3 / 78(3 \cdot 8)$ & $0 / 13(0)$ & Reference & & & \\
\hline $200-499 \mathrm{cell} / \mathrm{mm}^{3}$ & $25 / 78(32 \cdot 1)$ & $4 / 13(30 \cdot 8)$ & Not estimable & & & \\
\hline$\geqslant 500 \mathrm{cell} / \mathrm{mm}^{3}$ & $50 / 78(64 \cdot 1)$ & $9 / 13(69 \cdot 2)$ & Not estimable & & & \\
\hline Previous pregnancy & $78 / 237(32 \cdot 9)$ & $15 / 25(60)$ & $3 \cdot 1(1 \cdot 3-7 \cdot 3)$ & $0 \cdot 008$ & $2 \cdot 6(1 \cdot 1-6 \cdot 7)$ & $0 \cdot 04$ \\
\hline Previous pregnancy interruption & $90 / 237(38)$ & $9 / 25(36)$ & $0 \cdot 9(0 \cdot 4-2 \cdot 1)$ & $0 \cdot 85$ & & \\
\hline Previous spontaneous abortion & $27 / 237(11 \cdot 4)$ & $2 / 25(8)$ & $0 \cdot 7(0 \cdot 1-2 \cdot 5)$ & $0 \cdot 59$ & & \\
\hline PROM & $88 / 237(37 \cdot 1)$ & $7 / 25(28)$ & $0 \cdot 7(0 \cdot 2-1 \cdot 6)$ & $0 \cdot 36$ & & \\
\hline
\end{tabular}

MTCT, mother-to-child transmission; HBV, hepatitis B virus; HCV, hepatitis C virus; ART, antiretroviral therapy; cART, combined antiretroviral therapy; PROM, premature rupture of membranes.

*Variables with $P<0 \cdot 1$ in univariate analysis (decade of birth, age $\geqslant 32$ years, type of ART during pregnancy, antiretroviral prophylaxis intrapartum and previous pregnancy) were included in multivariable analysis. Timing of HIV diagnosis and ART prescription during pregnancy were excluded from multivariable analysis because of collinearity with the variable 'type of ART during pregnancy'.

$\dagger$ Percentages exclude unknown/missing results.

* Measurement closest to delivery. 
Table 3. Univariate and multivariable logistic regression analysis for severe SGA infants (newborn birth weight lower than 3 rd percentile for the gestational age $)^{\dagger}$

\begin{tabular}{|c|c|c|c|c|c|c|}
\hline & \multirow{2}{*}{$\begin{array}{l}\text { No severe SGA } \\
(N=207) \\
n / N(\%)\end{array}$} & \multirow{2}{*}{$\begin{array}{l}\text { Severe SGA } \\
(N=55) \\
n / N(\%)\end{array}$} & \multicolumn{2}{|c|}{ Univariate analysis } & \multicolumn{2}{|c|}{ Multivariable analysis* } \\
\hline & & & OR $(95 \% \mathrm{CI})$ & $P$ value & $\begin{array}{l}\text { Adjusted OR } \\
(95 \% \mathrm{CI})\end{array}$ & $P$ value \\
\hline Decade of birth & & & & $0 \cdot 11$ & & \\
\hline 1985-1994 & 98/207 (47-3) & $23 / 55(41 \cdot 8)$ & Reference & & & \\
\hline 1995-2004 & $50 / 207(24 \cdot 2)$ & $21 / 55(38 \cdot 2)$ & $1 \cdot 8(1-5 \cdot 2)$ & & & \\
\hline 2005-2014 & $59 / 207(28 \cdot 5)$ & $11 / 55(20)$ & $0 \cdot 8(0 \cdot 4-1 \cdot 7)$ & & & \\
\hline Age $\geqslant 32$ years & $67 / 207(32 \cdot 4)$ & $21 / 55(39 \cdot 2)$ & $1 \cdot 3(0 \cdot 7-2 \cdot 4)$ & $0 \cdot 41$ & & \\
\hline Ethnic group & & & & $0 \cdot 88$ & & \\
\hline Caucasian & $179 / 207(86 \cdot 5)$ & $48 / 55(87 \cdot 3)$ & Reference & & & \\
\hline Not Caucasian & 28/207 (13·5) & $7 / 55(12 \cdot 7)$ & $0 \cdot 9(0 \cdot 4-2 \cdot 2)$ & & & \\
\hline \multicolumn{7}{|c|}{ HIV risk factors (mode of transmission) } \\
\hline Injecting drugs use & $96 / 207(46 \cdot 4)$ & $31 / 55(56 \cdot 4)$ & $1 \cdot 5(0 \cdot 8-2 \cdot 7)$ & $0 \cdot 19$ & & \\
\hline Heterosexual intercourse & $84 / 207(40 \cdot 6)$ & $15 / 55(27 \cdot 3)$ & $0 \cdot 6(0 \cdot 3-1 \cdot 3)$ & $0 \cdot 07$ & $0 \cdot 8(0 \cdot 3-1 \cdot 6)$ & $0 \cdot 48$ \\
\hline MTCT & 2/207 (1) & $1 / 55(1 \cdot 8)$ & $1 \cdot 9(0 \cdot 1-20 \cdot 2)$ & $0 \cdot 62$ & & \\
\hline Other & $25 / 207(12 \cdot 1)$ & $8 / 55(14 \cdot 6)$ & $1 \cdot 2(0 \cdot 5-2 \cdot 8)$ & $0 \cdot 63$ & & \\
\hline \multicolumn{7}{|l|}{ Co-infection } \\
\hline HCV co-infection & $43 / 207(20 \cdot 8)$ & $17 / 55(30 \cdot 9)$ & $1 \cdot 7(0 \cdot 9-3 \cdot 3)$ & $0 \cdot 12$ & & \\
\hline HBV co-infection & $25 / 207(12 \cdot 1)$ & $7 / 55(12 \cdot 7)$ & $1 \cdot 1(0 \cdot 4-2 \cdot 5)$ & $0 \cdot 9$ & & \\
\hline HBV and HCV co-infection & $12 / 207(5 \cdot 8)$ & $4 / 55(7 \cdot 3)$ & $1 \cdot 3(0 \cdot 3-3 \cdot 8)$ & $0 \cdot 69$ & & \\
\hline Smoke & $36 / 207(17 \cdot 4)$ & $17 / 55(30 \cdot 9)$ & $2 \cdot 1(1 \cdot 1-4 \cdot 1)$ & $0 \cdot 03$ & $1 \cdot 8(0 \cdot 8-3 \cdot 7)$ & $0 \cdot 13$ \\
\hline Use of methadone or heroin & $22 / 207(10 \cdot 6)$ & $17 / 55(30 \cdot 9)$ & $3 \cdot 8(1 \cdot 8-7 \cdot 8)$ & $<0 \cdot 001$ & $3 \cdot 1(1 \cdot 4-6 \cdot 8)$ & $0 \cdot 007$ \\
\hline Timing of HIV diagnosis & & & & $0 \cdot 7$ & & \\
\hline Prior to pregnancy & $126 / 207(60 \cdot 9)$ & $35 / 55(63 \cdot 6)$ & Reference & & & \\
\hline During pregnancy & $40 / 207(19 \cdot 3)$ & $8 / 55(14 \cdot 6)$ & $0 \cdot 7(0 \cdot 3-1 \cdot 6)$ & & & \\
\hline At/after delivery & $41 / 207(19 \cdot 8)$ & $12 / 55(21 \cdot 8)$ & $1 \cdot 1(0 \cdot 5-2 \cdot 2)$ & & & \\
\hline ART at conception & $40 / 207(19 \cdot 3)$ & $5 / 55(9 \cdot 1)$ & $0 \cdot 4(0 \cdot 2-1)$ & $0 \cdot 06$ & $0 \cdot 4(0 \cdot 1-1 \cdot 3)$ & $0 \cdot 16$ \\
\hline ART prescription during pregnancy & $106 / 207(51 \cdot 2)$ & $26 / 55(47 \cdot 3)$ & $0 \cdot 9(0 \cdot 5-1 \cdot 5)$ & $0 \cdot 6$ & & \\
\hline Type of ART during pregnancy & & & & $0 \cdot 8$ & & \\
\hline No ART & 101/207 (48·8) & $29 / 55(52 \cdot 7)$ & Reference & & & \\
\hline Monotherapy/dual therapy & $36 / 207(17 \cdot 4)$ & $10 / 55(18 \cdot 2)$ & $1(0 \cdot 4-2 \cdot 1)$ & & & \\
\hline cART & $70 / 207(33 \cdot 8)$ & $16 / 55(29 \cdot 1)$ & $0 \cdot 8(0 \cdot 4-1 \cdot 6)$ & & & \\
\hline Antiretroviral prophylaxis intrapartum & $92 / 207(44 \cdot 4)$ & $25 / 55(45 \cdot 5)$ & $1(0 \cdot 6-1 \cdot 9)$ & 0.89 & & \\
\hline HIV-RNA load at delivery $(n=90)$ & & & & 0.51 & & \\
\hline Undetectable $(\leqslant 50 \mathrm{cp} / \mathrm{ml})$ & $47 / 71(66 \cdot 2)$ & $11 / 19(57 \cdot 9)$ & Reference & & & \\
\hline Detectable $(>50 \mathrm{cp} / \mathrm{ml})$ & $24 / 71(33 \cdot 8)$ & $8 / 19(42 \cdot 1)$ & $1 \cdot 4(0 \cdot 5-4)$ & & & \\
\hline $\mathrm{CD}^{+}$T-cell at delivery $(n=91)$ & & & & $0 \cdot 46$ & & \\
\hline$<200 \mathrm{cell} / \mathrm{mm}^{3}$ & $2 / 72(2 \cdot 8)$ & $1 / 19(5 \cdot 3)$ & Reference & & & \\
\hline $200-499$ cell $/ \mathrm{mm}^{3}$ & $11 / 72(29 \cdot 2)$ & $8 / 19(52 \cdot 6)$ & $0 \cdot 8(0 \cdot 06-17 \cdot 7)$ & & & \\
\hline$\geqslant 500$ cell $/ \mathrm{mm}^{3}$ & $49 / 72(68 \cdot 1)$ & $10 / 19(52 \cdot 6)$ & $0 \cdot 41(0 \cdot 04-9 \cdot 3)$ & & & \\
\hline Previous pregnancy & $74 / 207(35 \cdot 8)$ & $19 / 55(34 \cdot 6)$ & $0.9(0 \cdot 5-1 \cdot 8)$ & $0 \cdot 87$ & & \\
\hline Previous pregnancy interruption & $83 / 207(40 \cdot 1)$ & $16 / 55(29 \cdot 1)$ & $0 \cdot 6(0 \cdot 3-1 \cdot 2)$ & $0 \cdot 13$ & & \\
\hline Previous spontaneous abortion & $22 / 207(10 \cdot 6)$ & $7 / 55(12 \cdot 7)$ & $1 \cdot 2(0 \cdot 5-2 \cdot 9)$ & $0 \cdot 66$ & & \\
\hline PROM & $75 / 207(36 \cdot 2)$ & $20 / 55(36 \cdot 4)$ & $1(0 \cdot 5-1 \cdot 9)$ & 0.99 & & \\
\hline
\end{tabular}

SGA, small for gestational age; MTCT, mother-to-child transmission; HBV, hepatitis B virus; HCV, hepatitis C virus; ART, antiretroviral therapy; cART, combined antiretroviral therapy; PROM, premature rupture of membranes.

*Variables with $P<0 \cdot 1$ in univariate analysis (heterosexual intercourse as mode of transmission, smoke, use of methadone or heroin, ART at conception) were included in multivariable analysis.

$\dagger$ Percentages exclude unknown/missing results.

$\$$ Measurement closest to delivery. 
women were not virologically suppressed at delivery. Nevertheless, in the last decade only 15/65 (23.1\%) of the caesarean sections occurred in women not virologically suppressed or with a plasma HIV-RNA load not known at delivery. This suggests that in $76.9 \%$ of cases the caesarean section was not justified according to national and international guidelines. The procedure might have instead been due to the gynaecologists' clinical concern regarding the theoretical risk of HIV transmission associated with obstetrical interventions.

In our cohort, HIV vertical transmission rates gradually decreased during the first decade and then rapidly dropped between 1995 and 2000. No instances of MTCT occurred since 2000. This dramatic decrease in MTCT is consistent with data published in other European studies that reported a decrease in HIV vertical transmission rates from $15 \%$ to $25 \%$ in the early 1990 s to $<2 \%$ in recent years $[9,26,27]$. In the UK and Ireland, the rate of MTCT declined from $2 \cdot 1 \%$ in $2000-2001$ to $0 \cdot 46 \%$ in $2010-2011$ due to the widespread use of cART [3]. The zeroing of MTCT among HIV-infected pregnant women in our cohort since 2000 is a remarkable achievement, enabled by excellent improvements in HIV detection rates before or at least during early pregnancy, the extensive and routine use of cART among HIV-infected women and the use of elective caesarean sections in women not virologically suppressed.

In our study, preterm delivery rose substantially from $5.0 \%$ in the first decade to $17 \cdot 1 \%$ in recent years. The increase in preterm deliveries since the second decade may be related to ART, which has been routinely prescribed in pregnancy since 1994 [28]. In our cohort, only 45 women conceived on ART and 132 were on ART during pregnancy; 46 were receiving mono/dual therapy and 86 were receiving cART. Although the statistical power is low, our multivariable regression analysis revealed that preterm delivery was associated with maternal age and previous live births; no association with cART or other variables was noted. Other studies have investigated the association between preterm delivery and cART and noted heterogeneous results. A possible association between cART and prematurity was initially suggested in 1998 in a Swiss study that reported premature deliveries in 10 out of 30 women receiving cART [29]. Townsend et al. reported that cART was associated with a $1 \cdot 5$-fold increased risk of premature delivery compared with mono/dual therapy [30]. Cotter et al. reported a similar association, but limited to PI-containing regimens [31]. In contrast, Kourtis et al., in their meta-analysis, did not demonstrate an increased overall risk of premature delivery associated with the use of cART, even though the early initiation of cART during pregnancy appeared to confer a slightly increased risk of premature delivery [32]. Finally, the just-published PROMISE trial reported that preterm delivery was more frequent with zidovudine-based ART than with zidovudine monotherapy [33]. Discrepant results among different studies could derive from differences in population characteristics, the antiretroviral regimens used and the study periods. The ECS reported an association with the period of the study and increasing maternal age [34]. Although our findings derived from a single centre, they might concur to disprove the association between preterm delivery and cART. The increase in preterm delivery rates reported in recent years in our study may be related to advancing maternal age rather than cART use, considering that advanced maternal age is a known risk factor for preterm delivery in the general population [35]. Moreover, we found a previous live birth to be risk factor for preterm delivery, in accordance with the findings of an observational Italian study conducted by Liuzzi et al. [19]. The mechanisms underlying preterm delivery are complex and not completely understood. Nevertheless, an investigation of these mechanisms is beyond the objectives of this study.

The rate of severe SGA remained stable over the course of the study period. In our study, severe SGA was associated with the use of heroin or methadone, but not with other risk factors. Although cART has been suggested to be a possible risk factor for SGA, the relation between SGA and cART remains unclear. An increased risk of very low birthweight $(<1500 \mathrm{~g})$ has been reported in one study [36], but not in others $[27,31,36]$. Townsend et al. reported that cART-exposed infants were of lower birthweight than those exposed to mono/dual therapy, but the difference was not clinically significant [30]. Other than these studies, the association between SGA and chronic use of methadone during pregnancy stems from consolidated data [37-40]. The low statistical power of our study was unable to yield solid evidence for the absence of an association between preterm delivery or SGA and cART.

The strength of our study is represented by its long study period, which allowed us to describe the radical demographic and clinical changes occurring in HIVinfected pregnant women over the course of three 
decades. There were, however, a number of limitations to our study. First, the small number of patients in our cohort, which resulted in a low statistical power of our analysis, represents the major limitation. Second, we report a single-centre experience that included either women enrolled prospectively from ECS and women not enrolled in ECS or excluded women because of their presentation at delivery. Third, due to the retrospective design of the study, limited obstetrical data were available. Furthermore, immunological and virological data were available only for the last two decades and not for all patients. Finally, we included only women whose pregnancies ended in birth; we were accordingly unable to estimate changes in the miscarriage rate.

In conclusion, our study highlights the changing nature of the HIV-infected pregnant population. Above all, the increase in the median age of HIV-infected pregnant women could have an impact on neonatal outcomes, such as an increased risk of preterm delivery. The widespread use of cART prior to and during pregnancy made it possible to achieve the suppression of HIV-RNA load upon delivery in the majority of cases, with a meaningful contribution to the absolute decrease in MTCT. Nevertheless, in a 'real-life' setting of care there are still women showing up late in their pregnancies and with detectable HIV-RNA load at delivery. This situation persists not only among foreigners but primarily among Italian women. This fact suggests that it is important to encourage clinicians to engage in early HIV testing for all pregnant women and promote the routine use of cART during pregnancy to maintain a high level of clinical care for all women, who are pregnant or are planning a pregnancy. Not delivering vaginally represents a missed opportunity for HIV-infected women on cART with a suppressed HIV-RNA load and, according to international guidelines, caesarean sections should be restricted to women with late linkage-to-care and without virological suppression. In this regard, future research is necessary to address how earlier engagement in care among HIV-positive pregnant women can be promoted, to achieve viral suppression and the opportunity for HIV-positive pregnant women to deliver vaginally.

Finally, the retrospective nature of our investigation did not allow us to confirm previous studies pertaining to the missing association between cART and the increased risk of preterm delivery or severe SGA. With the changing nature of the HIV-infected female population and the introduction of new antiretroviral drugs as integrase strand transfer inhibitors, further research is needed - especially randomised, controlled clinical trials such as the PROMISE study [33] - to assess the risk of preterm delivery and SGA in different ethnic populations and in women receiving new antiretroviral regimens.

\section{SUPPLEMENTARY MATERIAL}

The supplementary material for this article can be found at https://doi.org/10.1017/S095026881700053X

\section{ACKNOWLEDGEMENTS}

This research received no specific grant from any funding agency, commercial or not-for-profit sectors. The authors wish to thank all HIV-1-infected pregnant women who participated in the study and medical and nursing staff at Infectious Diseases and Obstetrics and Gynaecology, IRCCS San Martino-IST, Genoa. They also wish to thank the staff at Hygiene Service for the important technical support in the laboratory testing. Finally, the authors wish to thank Dr Andrea Lisi for the review of the English language.

\section{CONTRIBUTOR MEMBERS OF GENOVA HIV NEONATAL AND PREGNANCY WORKING GROUP}

A. Calzi, A. Ferrazin, G. Mazzarello, F. Portunato, S. Ratto, R. Rosso, B. Wenda (Infectious Diseases Unit, IRCCS AOU San Martino-IST, University of Genoa, Genoa, Italy); C. Dentone (Infectious Diseases Unit, ASL-1 Imperiese, Sanremo (Imperia) Italy); G. Bentivoglio, L. Gabbi (Department of Obstetrics and Gynecology, IRCCS AOU San Martino-IST, Genoa, Italy); A. Loy (Infectious Diseases Unit, Istituto Giannina Gaslini, Genoa, Italy); M. Setti (Internal Medicine and Clinical Immunology Unit, IRCCS AOU San Martino-IST, Genoa, Italy).

\section{DECLARATION OF INTEREST}

None.

\section{REFERENCES}

1. World Health Organization. Programmatic update: use of antiretroviral drugs for treating pregnant women and preventing HIV infection in infants. (http://www.who.int/ hiv/PMTCT_update.pdf). Accessed 13 May 2016. 
2. Centers for Disease Control and Prevention. Enhanced perinatal surveillance-15 areas, 2005-2008. HIV Surveillance Supplemental Report 2011; 16(2). (http://www.cdc.gov/hiv/ pdf/statistics_2005_2008_HIV_Surveillance_Report_vol_16_ no2.pdf). Accessed 11 November 2016.

3. Townsend CL, et al. Earlier initiation of ART and further decline in mother-to-child HIV transmission rates, 2000-2011. AIDS 2014; 28: 1049-1057.

4. Istituto Superiore di Sanità. Dati HIV AIDS - Notiziario Istituto Superiore di Sanità (ISS) 2015; 28(9), Supplement 1. (http://www.iss.it/binary/ccoa/cont/HIV_ AIDS_DIC_2015.pdf). Accessed 18 March 2016.

5. Panel on Treatment of HIV-Infected Pregnant Women and Prevention of Perinatal Transmission. Recommendations for Use of Antiretroviral Drugs in Pregnant HIV-1-infected Women for Maternal Health and Interventions to Reduce Perinatal HIV Transmission in the United States. (http://aidsinfo.nih.gov/contentfiles/lvguidelines/PerinatalGL.pdf). Accessed 28 March 2016.

6. Watts DH, et al. Complications according to mode of delivery among human immunodeficiency virus-infected women with CD4 lymphocyte counts of $<$ or $=500 /$ microL. American Journal of Obstetrics \& Gynecology 2000; 183: 100-107.

7. Bulterys M, et al. Sexual behavior and injection drug use during pregnancy and vertical transmission of HIV-1. Journal of Acquired Immune Deficiency Syndromes 1997; 15: 76-82.

8. EUROSTAT news release 15 May 2015; International day of families. Women in EU gave birth to their first child at almost 29 years of age on average. (http://ec. europa.eu/eurostat/documents/2995521/6829228/3-13052015CP-EN.pdf/7e9007fb-3ca9-445f-96eb-fd75d6792965). Accessed 07 November 2016.

9. European Collaborative Study. HIV-infected pregnant women and vertical transmission in Europe since 1986. AIDS 2001; 15: 761-770.

10. EACS. European AIDS Clinical Society Guidelines Version 8.0 October 2015. (http://www.eacsociety.org/ files/2015_eacsguidelines_8_0-english_rev-20160124.pdf). Accessed 18 March 2016.

11. Società Italiana di Malattie Infettive e Tropicali (SIMIT). Linee Guida Italiane sull'utilizzo dei farmaci antiretrovirali e sulla gestione diagnostico-clinica delle persone con infezione da HIV-1, 2016 (http://www. salute.gov.it/imgs/C_17_pubblicazioni_2545_allegato.pdf). Accessed 3 January 2017.

12. Vayssière $\mathbf{C}$, et $\boldsymbol{a l}$. Fetal growth restriction and intrauterine growth restriction: guidelines for clinical practice from the French College of Gynaecologists and Obstetricians. European Journal of Obstetrics \& Gynecology and Reproductive Biology 2015; 193: 10-18.

13. Unterscheider $\mathbf{J}$, et al. Optimizing the definition of intrauterine growth restriction: the multicenter prospective PORTO Study. American Journal of Obstetrics and Gynecology 2013; 208: 290. e1-6.

14. Bruzzone B, et al. Impact of extensive HIV-1 variability on molecular diagnosis in the Congo basin. Journal of Clinical Virology 2010; 47: 372-375.
15. Agresti A. An Introduction to Categorical Data Analysis, 2nd edn. Hoboken: John Wiley \& Sons, 2007, pp. 357.

16. Fay MP, Proschan MA. Wilcoxon-Mann-Whitney or t-test? On assumptions for hypothesis tests and multiple interpretations of decision rules. Statistics Surveys 2010; 4: 1-39.

17. Peters L, Klein MB. Epidemiology of hepatitis $\mathrm{C}$ virus in HIV-infected patients. Current Opinion in HIV and AIDS 2015; 10: 297-302.

18. Townsend CL, et al. Low rates of mother-to-child transmission of HIV following effective pregnancy interventions in the United Kingdom and Ireland, 2000-2006. AIDS 2008; 22: 973-981.

19. Liuzzi G, et al. Pregnancy outcomes in HIV-infected women of advanced maternal age. HIV Clinical Trials 2013; 14: 110-119.

20. Aebi-Popp K, et al. Missed opportunities among HIVpositive women to control viral replication during pregnancy and to have a vaginal delivery. Journal of Acquired Immune Deficiency Syndromes 2013; 64: 58-65.

21. Huntington SE, et al. Predictors of pregnancy and changes in pregnancy incidence among HIV positive women accessing HIV clinical care at 13 large UK clinics. AIDS 2013; 27: 95-103.

22. Report ISTAT. Gravidanza, parto e allattamento al seno. Istituto Nazionale di Statistica (ISTAT) 2013. (http://www.istat.it/it/files/2014/12/gravidanza.pdf?title= Gravidanza $\% 2 \mathrm{C}+$ parto+e+allattamento+al+seno+-+09 $\% 2$ Fdic $\% 2 F 2014+-+$ Testo+integrale.pdf). Accessed 18 March 2016.

23. Aebi-Popp K, et al. National Guidelines for the prevention of mother-to-child transmission of HIV across Europe - how do countries differ? European Journal of Public Health 2013; 23: 1053-1058.

24. Kind C, et al. Prevention of vertical HIV transmission: additive protective effect of elective Cesarean section and zidovudine prophylaxis. Swiss Neonatal HIV Study Group. AIDS 1998; 12: 205-210.

25. The International Perinatal HIV Group. The mode of delivery and the risk of vertical transmission of human immunodeficiency virus type 1 -a meta-analysis of 15 prospective cohort studies. New England Journal of Medicine 1999; 340: 977-987.

26. Duong T, et al. Vertical transmission rates for HIV in the British Isles: estimates based on surveillance data. British Medical Journal 1999; 319: 1227-1229.

27. European Collaborative Study. Mother-to-child transmission of HIV infection in the era of highly active antiretroviral therapy. Clinical Infectious Diseases 2005; 40: 458-465.

28. Connor EM, et al. Reduction of maternal-infant transmission of human immunodeficiency virus type 1 with zidovudine treatment. New England Journal of Medicine 1994; 331: 1173-1180.

29. Lorenzi P, et al. Antiretroviral therapies in pregnancy: maternal, fetal and neonatal effects. Swiss HIV Cohort Study, the Swiss Collaborative HIV and Pregnancy Study, and the Swiss Neonatal HIV Study. AIDS 1998; 12: F241-F247. 
30. Townsend CL, et al. Antiretroviral therapy and premature delivery in diagnosed HIV-infected women in the United Kingdom and Ireland. AIDS 2007; 21: 10191026.

31. Cotter AM, et al. Is antiretroviral therapy during pregnancy associated with an increased risk of preterm delivery, low birth weight, or stillbirth? The Journal of Infectious Diseases 2006; 193: 1195-1201.

32. Kourtis AP, et al. Use of antiretroviral therapy in pregnant HIV-infected women and the risk of premature delivery: a meta-analysis. AIDS 2007; 21: 607-615.

33. Fowler MG, et al. Benefits and risks of antiretroviral therapy for perinatal HIV prevention. New England Journal Medicine 2016; 375: 1726-1737.

34. European Collaborative Study. Exposure to antiretroviral therapy in utero or early life: the health of uninfected children born to HIV-infected women. Journal of Acquired Immune Deficiency Syndromes 2003; 32: 380-387.

35. Hoffman MC, et al. Pregnancy at or beyond age 40 years is associated with an increased risk of fetal death and other adverse outcomes. American Journal of Obstetrics \& Gynecology 2007; 196: e11-13.

36. Tuomala RE, et al. Antiretroviral therapy during pregnancy and the risk of an adverse outcome. New England Journal of Medicine 2002; 346: 1863-1870.

37. Pritham UA, Troese M, Stetson A. Methadone and buprenorphine treatment during pregnancy. What are the effects? Nursing for Women's Health 2007; 11: 558-567.

38. Mozurkewich EL, Rayburn WF. Buprenorphine and methadone for opioid addiction during pregnancy. Obstetrics and Gynecology Clinics of North America 2014; 41: 241-253.

39. Kakko J, Heilig M, Sarman I. Buprenorphine and methadone treatment of opiate dependence during pregnancy: comparison of fetal growth and neonatal outcomes in two consecutive case series. Drug and Alcohol Dependence 2008; 96: 69-78.

40. Brogly SB, et al. Prenatal buprenorphine versus methadone exposure and neonatal outcomes: systematic review and meta-analysis. American Journal of Epidemiology 2014; 180: 673-686. 